\title{
TÚNEL HIDROCINEMÁTICO - SISTEMA S-PIV-3D - VELOCIMETRIA ESTEREOSCÓPICA POR IMAGENS DE PARTÍCULAS
}

\author{
WATER KINEMATIC TUNNEL - S-PIV-3D SYSTEM - \\ STEREOSCOPIC PARTICLE IMAGE VELOCIMETRY
}

\author{
Marcos Rogério Szeliga ${ }^{1}$, Woodrow Nelson Lopes Roma ${ }^{2}$ \\ ${ }^{1}$ Universidade Estadual de Ponta Grossa, Departamento de Engenharia Civil \\ <marcosrs@uepg.br> \\ 2 Universidade de São Paulo, Escola de Engenharia de São Carlos, Departamento de \\ Hidráulica e Saneamento.
}

\begin{abstract}
RESUMO
A velocimetria por imagem de partículas (particle image velocimetry - PIV) é uma técnica recente de medições em escoamentos por meio de uma metodologia não intrusiva. Com base na técnica PIV, foi desenvolvido o sistema S-PIV-3D, uilizando princípios semelhantes aos sistemas convencionais com a inclusão de algumas características exclusivas, como o método óptico de aquisição de imagens e o processo de calibragem do sistema de coordenadas, levando à obtenção de dados tridimensionais com o uso de apenas uma câmera convencional. Os resultados consistem na obtenção de campos de velocidades tridimensionais com produção de gráficos interativos, imagens e ferramentas de visualização. Com esses resultados, o sistema viabilizou a aplicação da técnica em laboratórios didáticos. Foi desenvolvido um software específico e um protótipo com características de adaptação às mais diversas situações de medição, especialmente aquelas que envolvem a demonstração do comportamento cinemático do escoamento na interação com modelos sólidos, como por exemplo contornos comuns em estudos hidráulicos, tais como cilindros, orifícios, comportas, soleiras e vertedouros. Para aplicação em laboratórios didáticos, foi desenvolvido o túnel hidrocinemático, equipamento que consiste em uma bancada de ensaios com produção de escoamento controlado e proporciona condições para estudos e visualização das características dos escoamentos com a aplicação do sistema S-PIV-3D. Este trabalho, por meio da descrição desse sistema, vem demonstrar a possibilidade de implantação de ferramentas avançadas em laboratórios didáticos por meio de desenvolvimento próprio e de baixo custo.
\end{abstract}

Palavras-chave: PIV. Velocimetria por imagens de partículas. Visão computacional. Métodos ópticos de medição. Diagnóstico do escoamento. 


\begin{abstract}
The Particle Image Velocimetry - PIV - is a recent technique for flow measurements through a non-intrusive methodology. The S-PIV-3D System was developed based on the PIV technique. In this system, similar principles to conventional systems were used with the addition of exclusive features, such as the optical method of image acquisition and the calibration process of the coordinate system leading to three-dimensional data using a single conventional camera. The results consist on three-dimensional velocity fields with production of interactive graphics, images and visualization tools. With these results the system enabled the application of the technique in teaching laboratories. A specific software was developed and a prototype with adaptation characteristics to a variety of measurement situations, especially those involving the demonstration of the kinematic behavior of the flow and its interaction with solid models, for example, common hydraulic studies, such as flow through cylinders, holes, bottom gates, sills, spillways and others. In order to be used in a teaching laboratory a water kinematic tunnel was developed. The equipment consists on an apparatus with flow production and provides controlled conditions for studies and visualization of the flow features with the application of the S-PIV-3D system. The description of this system demonstrates the possibility of deployment of advanced tools in didactics laboratories through self-development and low cost.
\end{abstract}

Keywords: PIV - Particle Image Velocimetry. Computational vision. Optical methods of measurement. Flow diagnosis.

\section{INTRODUÇÃO}

A crescente acessibilidade aos dispositivos de automação, controle computadorizado, aquisição e processamento de imagens torna viável o desenvolvimento de equipamentos para modernização de laboratórios didáticos de ensino de engenharia. $\mathrm{O}$ Brasil tem um grande déficit com relação ao reequipamento dos laboratórios de ensino e sua inclusão no atual contexto tecnológico, que sobremaneira envolve a informação digital, seu processamento e obtenção de resultados. O ensino de engenharia, mantendo sua concepção didática, tem que fornecer subsídios para acompanhar os meios contemporâneos de integração tecnológica. Nesse contexto, por meio de um projeto de pesquisa intitulado "Velocimetria de partículas por visão estereoscópica: Sistema S-PIV-3D", em conjunto com outro projeto, intitulado "Túnel hidrocinemático: Aplicação do sistema S-PIV-3D", o desenvolvimento de tecnologia baseada no conceito da velocimetria óptica incorporada nos sistemas PIV foi proposto e realizado, culminando em softwares e equipamentos com finalidades didáticas e de pesquisa. Esse desenvolvimento veio compor o preenchimento de uma lacuna verificada quando se compara a estrutura laboratorial nas instituições nacionais com instituições similares dos países desenvolvidos.

A velocimetria por imagem de partículas (particle image velocimetry - PIV) é uma tecnologia recente para obtenção de dados acerca do campo de velocidades em escoamentos. Suas primeiras aplicações comerciais datam de 1997 (LAVISION, 2006). A tecnologia utiliza o processamento digital das imagens produzidas no escoamento por um feixe laser pelo qual as partículas em suspensão são iluminadas, sendo fotografadas no intervalo de dois pulsos do feixe laser em sincronia com a aquisição da imagem. A distribuição das partículas é registrada em quadros sucessivos de um arquivo digital de imagens. Os sistemas convencionais utilizam uma câmera para aquisição de imagens $2 \mathrm{D}$ em escoamentos bidimensionais e duas câmeras sincronizadas para imagens $3 \mathrm{D}$ de escoamentos em que a terceira componente da velocidade tenha papel relevante. Com a utilização de apenas uma câmera é 
possível a obtenção de dados no plano perpendicular ao eixo de focagem da câmera. O uso de duas câmeras com aquisição simultânea possibilita a obtenção de coordenadas tridimensionais após a associação dos planos de focagem. As imagens processadas em software específico conduzem a dados geométricos que registram a distribuição de velocidades na área pesquisada por meio da obtenção do deslocamento das partículas na unidade de tempo. Os resultados obtidos são representados por meio de gráficos de distribuição vetorial no sistema de coordenadas, incluindo isolinhas de velocidades e vorticidades e linhas de corrente.

Os projetos de pesquisa indicados anteriormente envolveram o estudo e a aplicação de alternativas na geração, captura e processamento das imagens e se fundamentou basicamente em cinco itens:

- desenvolvimento do software de processamento de imagens utilizando a linguagem MatLab, tomando partido dos recursos matemáticos e de visualização proporcionados pela linguagem de programação;

- desenvolvimento de um estereoscópio ajustável que, em conjunto com apenas uma câmera digital, viabilizou a aquisição de imagens com os dados adequados para a definição tridimensional a partir de dois pontos de vista distintos;

- desenvolvimento dos dispositivos de calibração do sistema de coordenadas, por meio do quais foi possível estabelecer a conversão das medidas obtidas nas imagens para as medidas reais, operar a correção da distorção óptica gerada na aquisição das imagens e proporcionar ampla liberdade na instalação de equipamentos;

- utilização de uma iluminação por uma bateria de até seis unidades laser industriais de $3 \mathrm{~mW}$ com feixe em linha, de acordo com as necessidades de cobertura da região de medição, e estabelecimento de condições para aquisição de imagens em escoamentos de baixa velocidade, com custo reduzido de equipamentos;

- uso de uma câmera digital convencional de alta definição objetivando a obtenção de um protótipo de fácil instalação em qualquer laboratório de medição.
A questão central a nortear o desenvolvimento dos projetos estava em estabelecer os princípios de atuação na tecnologia baseados em requisitos para atendimento de medições voltadas para a demonstração do escoamento. Nesse sentido, a pesquisa voltou-se para a simplificação de métodos e dispositivos no intento de proporcionar a instalação do equipamento com baixo custo de implantação e operação.

O software para processamento das imagens e obtenção de campos de velocidades no escoamento foi denominado S-PIV-3D. Esse programa, elaborado com a linguagem MatLab, compreende uma etapa de calibração na qual, com o auxílio de uma malha predefinida e fotos de calibração, são obtidos os parâmetros de uma função de transformação de coordenadas das imagens para um sistema real de coordenadas. Outra etapa, após a calibração, envolve o processamento de imagens do escoamento. Esta etapa utiliza arquivos de imagens no formato audio video interleave (AVI) produzidos na aquisição para, por meio do algoritmo de correlações cruzadas normalizadas, obter o deslocamento das partículas fotografadas. Os deslocamentos divididos pela unidade de tempo, lançados nas células (áreas de interrogação) de uma malha produzida sobre a região de estudo conduzem aos vetores-velocidade. Este campo de velocidades tem seus valores calculados para cada quadro do filme de aquisição e é possível sua visualização em qualquer ângulo e a verificação de dados numéricos em qualquer instante.

Para estudos de campos tridimensionais, os sistemas PIV convencionais empregam duas ou mais câmeras de aquisição de imagens que atuam eletronicamente sincronizadas. O sistema S-PIV-3D utilizou um dispositivo óptico para eliminar a necessidade da segunda câmera, obtendo imagens oriundas de pontos de vista distintos por meio de um estereoscópio. Este dispositivo, utilizando espelhos de face frontal e um prisma de reflexão, foi desenhado de modo a flexibilizar o posicionamento e se adaptar adequadamente aos requisitos. A imagem resultante consiste em dois campos que dividem o quadro de imagem e, desta forma, tem-se uma aquisição naturalmente simultânea.

Para relacionar as imagens com o espaço real, foi desenvolvido um processo de calibração. Esse 
processo envolve a obtenção de parâmetros por meio de fotos de calibração obtidas pela própria câmera de aquisição acoplada ao estereoscópio na posição definitiva de operação. As fotos retratam uma placa com uma malha de calibração com dimensões predefinidas e posição, em relação ao sistema real de coordenadas, também predefinida. O posicionamento da malha é controlado por um dispositivo de suporte especialmente projetado para o fim de calibração. As fotos são obtidas de maneira padronizada e seu processamento estabelece uma relação entre as imagens obtidas e a forma da malha, definindo parâmetros de calibração usados em uma função de transformação de coordenadas (SZELIGA; ROMA, 2009).

Os sistemas convencionais utilizam iluminação laser pulsada em sincronia com a abertura dos obturadores das câmeras. Isso decorre da necessidade de máxima precisão na estipulação do intervalo de tempo entre a aquisição de uma imagem e a seguinte e para evitar o efeito blurring (espalhamento da luz ocasionando traços luminosos nos escoamentos mais rápidos). Entretanto, adotar esta solução existe implica no uso de equipamentos especiais. Assim, com a filosofia do desenvolvimento de um modelo que se caracterize pela simplicidade de sua instalação e o baixo custo de implantação, procuraram-se soluções para atender satisfatoriamente aos requisitos de medição, com usoda temporização caracterizada pela velocidade de aquisição dos quadros de imagem. Aumentando-se o número de medições sucessivas, os quadros do filme de aquisição, os deslocamentos médios tendem a convergir para o valor exato ocorrido no intervalo de tempo padrão da aquisição. Com a adoção da redução do tempo de exposição para captura de imagens, obteve-se uma minimização do efeito blurring.

Medições com o sistema S-PIV-3D foram realizadas no túnel hidrocinemático. Este equipamento consiste em uma bancada de ensaios com produção de escoamento controlado em uma variada gama do número de Reynolds, realizando ensaios de escoamentos em torno de corpos sólidos. Os resultados obtidos consistem em campos de velocidades em torno e a jusante dos modelos sólidos e correspondem a gráficos bidimensionais e tridimensionais compostos por distribuição de vetores e linhas de corrente. Esses gráficos podem ser manipulados interativamente de modo a se observar por qualquer ângulo, obter detalhes específicos e extrair dados numéricos. Uma visualização sucessiva de gráficos pode ser usada para verificação do comportamento cinemático do escoamento no espaço e no tempo. O túnel foi projetado para proporcionar um escoamento controlado e condicionado. Foram utilizadas estruturas de dissipação de turbulência na recirculação do circuito por motobomba. Na região de medição, as paredes são de acrílico para prover as necessidades de visualização e acessibilidade do sistema S-PIV-3D. A configuração do equipamento pode ser observada na Figura 1.

Figura 1 - Túnel Hidrocinemático

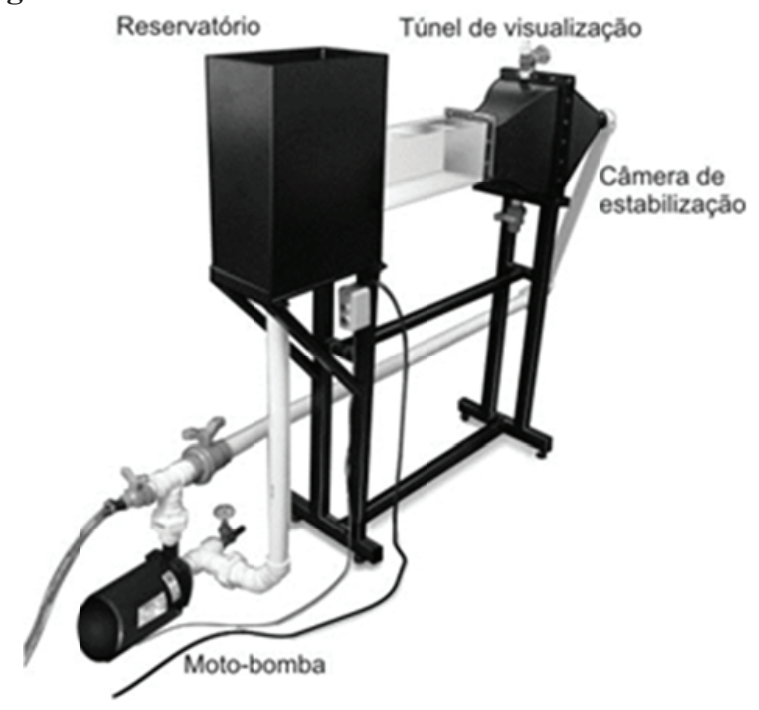

\section{REVISÃO BIBLIOGRÁFICA}

$\mathrm{O}$ atual desenvolvimento da técnica PIV, em nível mundial, deve-se aos recentes avanços no processamento digital e na capacidade de aquisição de imagens, especialmente as tecnologias baseadas em dispositivos charge coupled device (CCD) ou complementary metal-oxide semiconductor (CMOS). Os sistemas PIV convencionais utilizam uma câmera para imagens 2D em medições de escoamentos bidimensionais e duas ou mais câmeras sincronizadas para obtenção de dados em três dimensões, em escoamentos em que a terceira componente da velocidade tenha papel relevante (DANTEC DYNAMICS, 2005).

Uma instalação experimental de um sistema PIV consiste, tipicamente, de vários subsistemas. Conforme Raffel e colaboradores (2007), na maioria 
das aplicações partículas traçadoras, que devem ser adicionadas ao escoamento, são iluminadas e seu posicionamento é registrado entre um curto intervalo de tempo. Normalmente, a iluminação de faz por emissão laser em lâmina, abrangendo a área de interesse de medição. O registro de imagens por câmeras convencionais ou digitais pode ocorrer em um único frame ou uma seqüência de frames. O intervalo de tempo de exposição na aquisição da imagem deve ser pequeno o suficiente para congelar o movimento das partículas e evitar o efeito blurring.

O deslocamento das imagens das partículas é determinado por meio de técnicas PIV com utilização de metodologias de pós-processamento. Para a avaliação do campo de velocidades, um par de imagens PIV é dividido em pequenas subáreas chamadas de áreas de interrogação. O vetor deslocamento local para as imagens das partículas é determinado para cada área de interrogação por meio de métodos estatísticos - autocorrelação ou correlação cruzada. Assume-se que todas as partículas dentro de uma área de interrogação se movam de maneira relativamente homogênea entre as áreas de interrogação da primeira e da segunda imagem (RAFFEL et al., 2007).

Uma das características dos sistemas PIV é o pré-processamento. Muitas vezes, as imagens precisam de tratamento para melhorar suas características e se adaptar aos algoritmos de autocorrelação ou correlação cruzada. Uma das técnicas de pré-processamento que podem conduzir a melhores resultados é a suavização gaussiana, evidenciando as partículas na imagem e proporcionando melhores condições para os algoritmos de correlação (ELSINGA et al. 2005a).

A técnica PIV, em nível mundial, já compreende sistemas amplamente consolidados, seu emprego tem demonstrado excelentes resultados nas mais diferentes situações que envolvem o escoamento dos fluidos. Na pesquisa de base, podem ser citados numerosos pesquisadores e, a título de exemplo, seguem algumas referências.

Urban e Mungal (2001) aplicaram a técnica PIV em túnel de vento para obtenção de campos de velocidades bidimensionais em escoamento de alta velocidade - número de Mach até 2,25 e número de Reynolds até $10^{6}$. Yang e Rockwell (2002), utilizando um sistema PIV com duas câmeras de alta reso- lução, estudaram as interações de ondas na superfície livre com um cilindro vertical caracterizando a estrutura do escoamento em termos de velocidade instantânea e vorticidade. Smith e Glazer (2002) investigaram o escoamento de jatos de ar através de orifícios empregando um sistema PIV com câmeras de alta resolução - $10 \mathrm{MP}$, exposição de $1 \mu \mathrm{s}$, iluminação com um par de lasers NdYAg e produção de um feixe com espessura e largura reguláveis. Tao e colaboradores (2002), utilizando um sistema de holographic particle image velocimetry (HPIV), obtiveram a distribuição tridimensional de velocidades no escoamento turbulento em um tubo de seção quadrada com número de Reynolds da ordem de $1,2 \cdot 10^{5}$. Cicca e colaboradores (2002) investigaram a turbulência na camada de escoamento junto à parede de contorno sujeita a perturbações externas. No estudo, empregaram o processamento de imagens do sistema PIV com áreas de interrogação de 64x64 pixels e o algoritmo de correlação cruzada na definição dos deslocamentos. Na área de instrumentação aplicada, Mills e colaboradores (2003) desenvolveram um túnel de escoamento de água em que aplicaram ensaios com utilização das medições PIV em torno de cilindros retangulares. Nas medições, obtiveram resultados que variaram em menos de $0,5 \%$ quando comparados com os valores obtidos com a técnica de anemômetro de fio quente. Foram adicionadas microesferas de vidro banhadas em prata para atuarem como partículas traçadoras com uma densidade que permitia proximidade à flutuação neutra. A iluminação ocorreu por meio de um laser argon-ion - 6W, feixe contínuo, mas com pulsação controlada computacionalmente para produção de três pulsos na aquisição da imagem. No processamento das imagens, foi utilizado o algoritmo de autocorrelação e técnicas de remoção de ruídos. Com aplicação da técnica para visualização tridimensional do escoamento (stereoscopic particle image velocimetry), Alkislar e colaboradores (2003) investigaram experimentalmente escoamentos formados por jatos em regime não permanente. As medições demonstraram a evolução espacial e temporal do jato com alta fidelidade, além de apresentar os campos de velocidades e vorticidades. A aquisição ocorreu com uma câmera de resolução $1008 \times 1018$ pixels a uma taxa de 30 fps. Foi usado um pulsador laser com exposição entre 1 e 1,5 $\mu \mathrm{s}$. 
Dong e colaboradores (2005) combinaram a técnica PIV com simulações numéricas para o estudo do escoamento junto à parede de um cilindro, mostrando a influência do número de Reynolds na estrutura do escoamento. Pu e Meng (2005) introduziram inovações na técnica de holographic particle image velocimetry (HPIV) com visualizações de estruturas de vórtices por meio de isosuperfícies de vorticidade. Sengupta e colanboradores (2007) estudaram o escoamento incompressível e acelerado em torno de um aerofólio. Realizaram medições com a técnica PIV e, utilizando a simulação computacional com a equação de Navier-Stokes, estabeleceram comparações entre os resultados.

Em agosto de 2009, a Universidade de Monash, Melbourne, Austrália, promoveu o 8th International Symposium on Particle Image Velocimetry, que sucedeu às edições dos encontros científicos realizados em Fukui, Japão (1995 e 1997), Santa Barbara, Estados Unidos (1999), Göettingen, Alemanha (2001), Busan, Coreia (2003), Pasadena, Estados Unidos (2005) e Roma, Itália (2007).

\section{METODOLOGIA}

O sistema S-PIV-3D foi desenvolvido para se incluir na tecnologia de medição por imagens, apresentando algumas alternativas para implantação, operação e obtenção de resultados que o tornam acessível para laboratórios de demonstração das características do escoamento. Inicialmente, pode-se citar o processo de aquisição de imagens, que utiliza um dispositivo óptico para permitir a manipulação tridimensional. Um conjunto de espelhos planos e um prisma de faces espelhadas acoplados conforme uma geometria apropriada facilitou a aquisição de imagens simultâneas segundo pontos de vista distintos, o que é imprescindível para obtenção de dados tridimensionais. Com esse dispositivo óptico, um modelo de estereoscópio, foi possível a utilização de apenas uma câmera para aquisição de imagens. A produção de imagens utiliza módulos de emissão laser industriais com $3 \mathrm{~mW}$ de potência, aparelhos de baixo custo e operação contínua, sem pulsação em sincronia com a aquisição. Na Figura 2, pode-se observar a configuração estabelecida com o posicionamento da câmera diante do arranjo dos suportes de espelhos, com seus posicionadores de ajuste fino que constituem o estereoscópio. Pode-se notar, em função do caminho óptico estabelecido, o consequente compartilhamento do quadro de imagem decorrente do arranjo estereoscópico. Inclui-se, ainda, a disposição para iluminação por meio dos módulos laser com seus respectivos suportes de ajuste, acrescentando-se que podem ser utilizados diversos conjuntos de iluminadores de acordo com as necessidades. A Figura 3 apresenta o sistema S-PIV-3D instalado para ensaios no túnel hidrocinemático.

Figura 2 - Sistema S-PIV-3D: disposição dos elementos de produção e aquisição de imagens

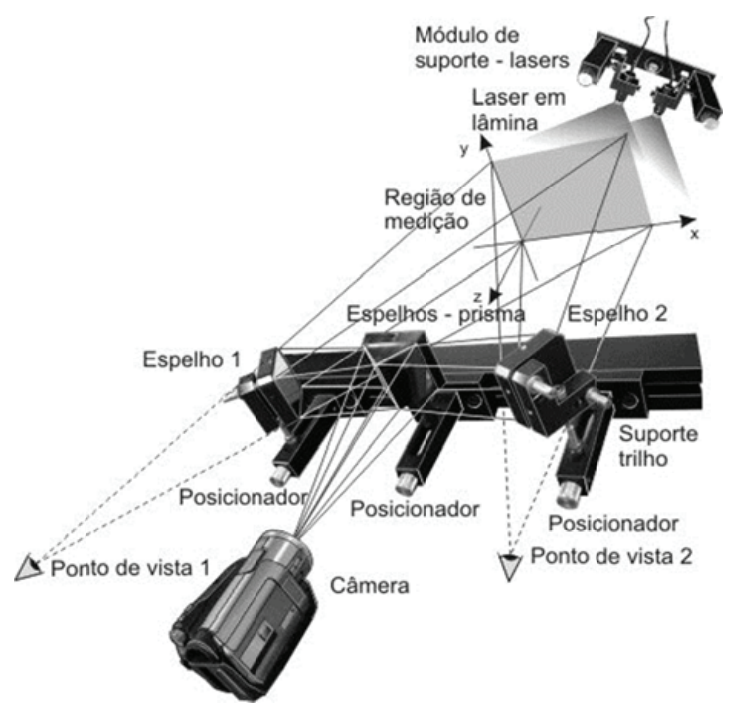

Figura 3 - SistemaS-PIV-3D e túnel hidrocinemático

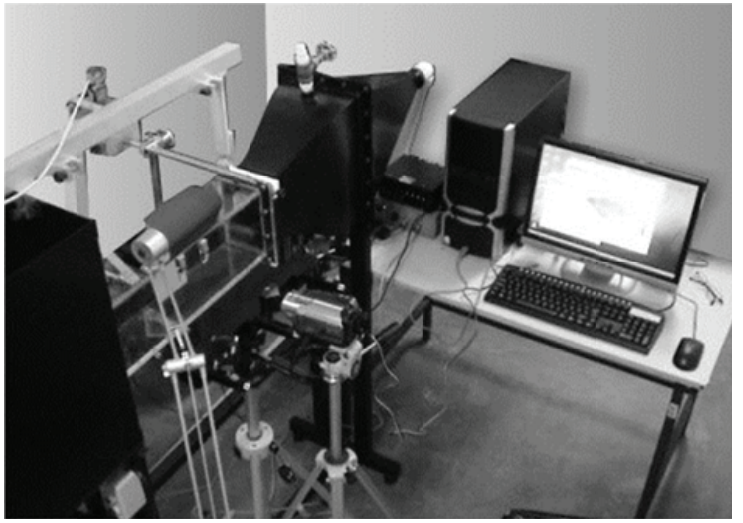

Na Figura 3, pode-se observar o dispositivo acima do túnel, que corresponde ao instrumento de calibração do sistema. Esse instrumento possibilita a liberdade de posicionamento dos dispositivos de aquisição de imagens e se fundamenta em uma relação entre imagem e objeto fotografado que se preserva durante a consecução de ensaios. Essa relação guarda fatores que englobam todas as variáveis que 
Figura 4 - Dispositivo de calibração e exemplo de imagem do grid

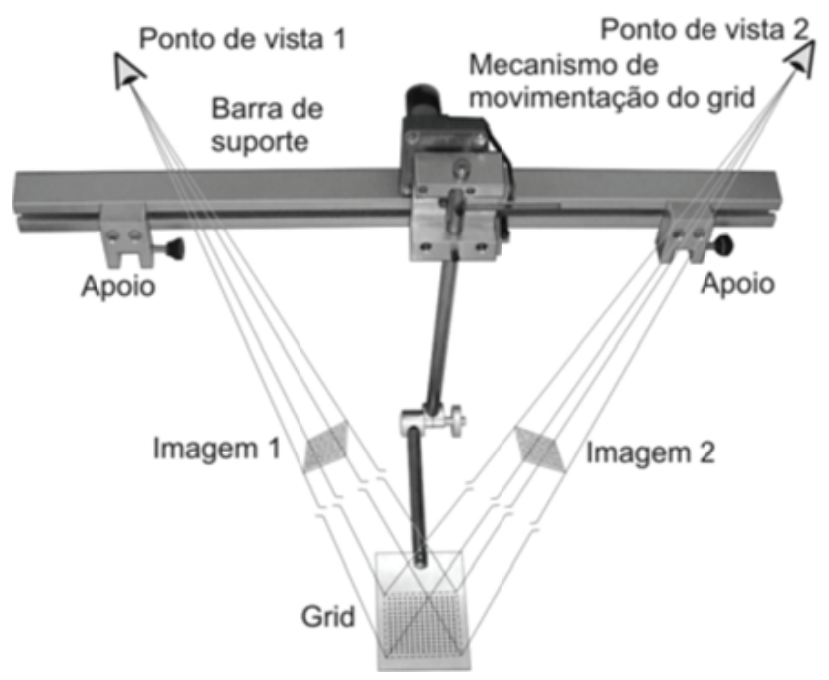

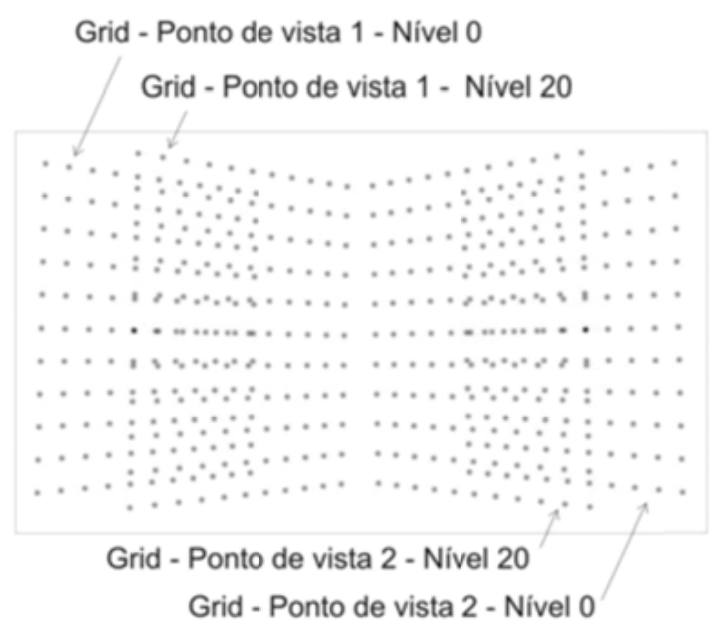

entre as imagens comparadas. Esses coeficientes podem atingir valores entre 1 (um positivo), quando a imagem pesquisada é exatamente igual ao recorte, e -1 (um negativo), quando a imagem pesquisada é exatamente inversa ao recorte. Aplicando-se esta formulação para a velocimetria por imagens de partículas entre duas imagens sucessivas, o que se determina é o deslocamento de um quadro que, recortado da primeira imagem, melhor se encontra na segunda imagem. Como existem sensíveis diferenças entre primeira e segunda imagens, o algoritmo vai determinar a melhor aproximação, retornando um coeficiente máximo (pico de correlação). Como o algoritmo pode retornar uma posição com precisão de apenas um pixel, ainda é realizada uma operação baseada na curva gaussiana para a aproximação subpixel. Com a Figura 5, ilustra-se o processo de correlação e determinação do vetor deslocamento.

Figura 5 - Algoritmo de correlação cruzada

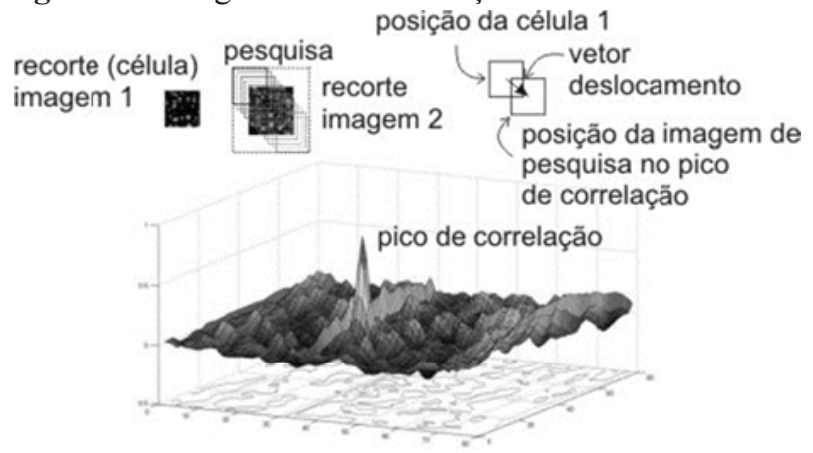


Figura 6 - Reconstrução do espaço tridimensional a partir das imagens planas
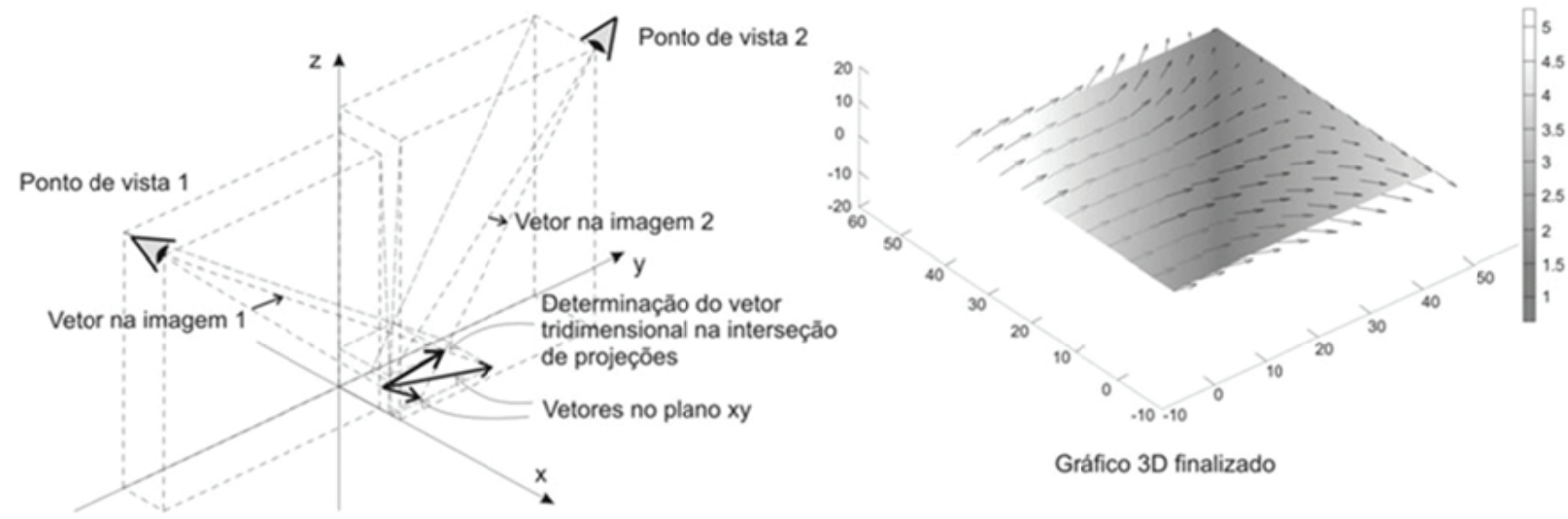

Gráfico 3D finalizado

Após as operações sobre as imagens de cada ponto de vista, têm-se duas malhas de vetores velocidade. As coordenadas estão relacionadas ao espaço projetivo, ou seja, com suas coordenadas em pixels sobre as imagens de aquisição. Neste ponto, é aplicada a transformação de coordenadas, obtendo-se malhas de vetores com valores relacionados ao sistema real de coordenadas. Com a associação das malhas, orientadas segundo os eixos de tomada de cena, procede-se à reconstrução dos vetores velocidade tridimensionais por meio das interseções das projeções, como pode ser observado no esquema mostrado na Figura 6.

O software desenvolvido com a linguagem Matlab e suas inerentes facilidades matemáticas também contém todos os requisitos para as análises e inferências decorrentes dos resultados obtidos com o sistema PIV-S. Utilizando interfaces gráficas com o usuário - graphic user interface (GUI) -, ele estabelece uma sequência de operações que se habilitam à medida que as tarefas necessárias para operar as imagens são cumpridas. E é composto por uma janela principal com a qual se estabelece o controle sobre diversas outras janelas de visualização de resultados, as quais são apresentadas à medida que as operações se realizam de maneira intuitiva. Ocorrem resultados gráficos em janelas adicionais que apresentam malhas de vetores, deslocamentos, médias e tendências. A Figura 7 reproduz a tela principal na qual se pode verificar a disponibilidade de comandos para operar as imagens de calibração, processar o arquivo de vídeo, salvar e também acessar resultados processados anteriormente.

Por meio de uma série de ensaios, foi definida a padronização de procedimentos. Os ensaios foram realizados no túnel hidrocinemático, concebido para

Figura 7 - Reprodução da tela principal do programa computacional S-PIV-3D

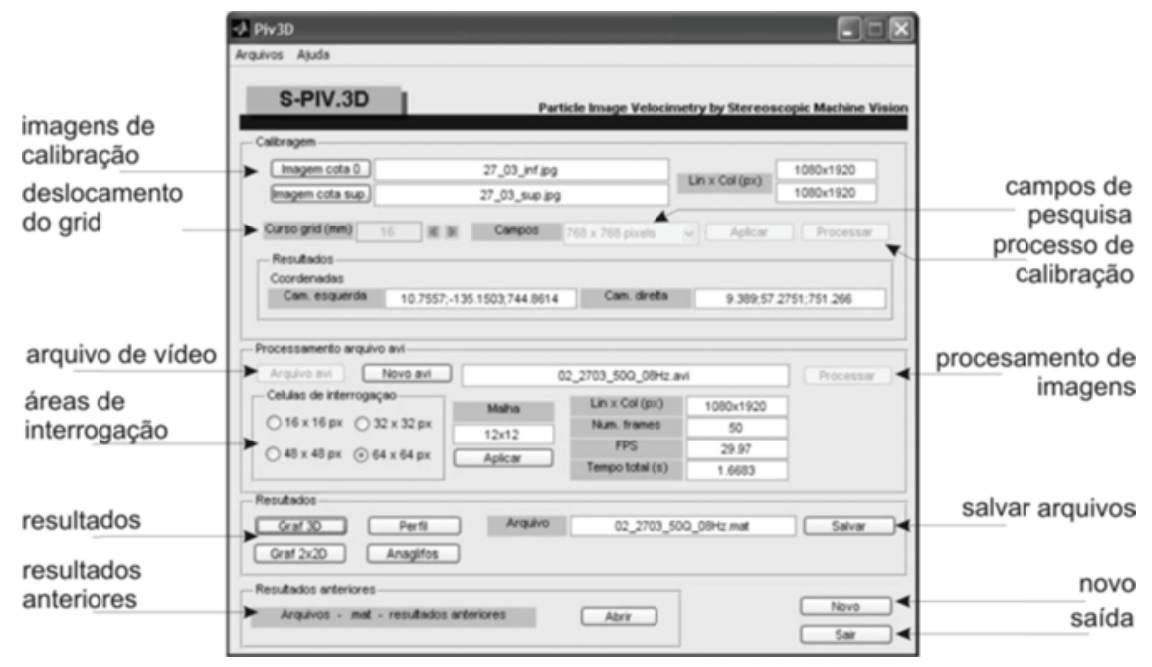

Publ. UEPG Exact Earth Sci., Agr. Sci. Eng., Ponta Grossa, 16 (2): 63-74, dez. 2010 
operar escoamentos em torno de modelos sólidos e se compor como um equipamento fundamental em laboratório didático de hidráulica. Com a Figura 8 se mostra, esquematicamente, o funcionamento interno do túnel e com a Figura 9, algumas fases do projeto.

Figura 8 - Esquema de funcionamento do túnel hidrocinemático

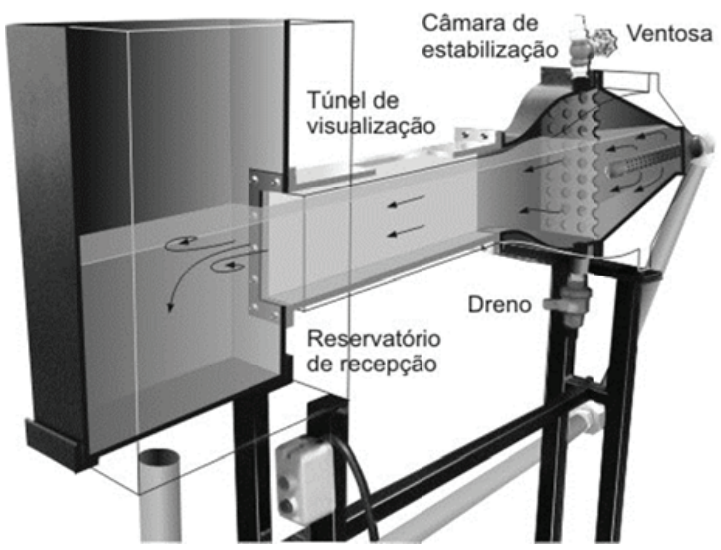

Figura 9 - Fases do projeto do túnel hidrocinemático

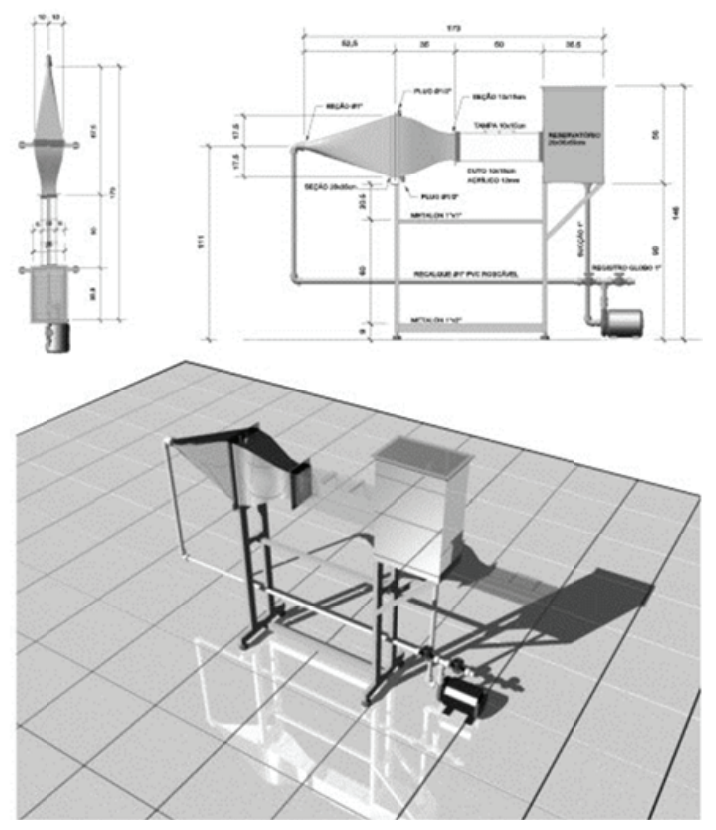

\section{RESULTADOS}

Nos resultados, por meio do programa computacional S-PIV-3D, pode-se observar a distribuição de vetores quadro a quadro; obter valores de velocidade, direções, médias; e perceber as características do escoamento por meio da visualização particular em determinado ponto ou região. De acordo com as características do escoamento, podem ser estudados planos bidimensionais de distribuição de velocidades, verificada a interação com o contorno e estabelecidas comparações com as medições tridimensionais, aprofundando as inferências acerca do campo de velocidades.

Os ensaios consistiram em medições bidimensionais e tridimensionais em torno e a jusante de modelos sólidos. A seguir, são apresentados alguns exemplos de ensaios e resultados obtidos com a aplicação do sistema S-PIV-3D no túnel hidrocinemático.

\subsection{Escoamento em torno de um cilindro.}

Neste ensaio, foi usado um cilindro horizontal com 25 mm de diâmetro acoplado entre as paredes do túnel. A lâmina laser foi direcionada verticalmente, com emissão distribuída de modo a evitar sombreamentos. As velocidades de operação ficaram em torno de $10 \mathrm{~cm} / \mathrm{s}$. A instalação deste ensaio está representada na Figura 10.

Figura 10 - Ensaio de escoamento em torno de um cilindro

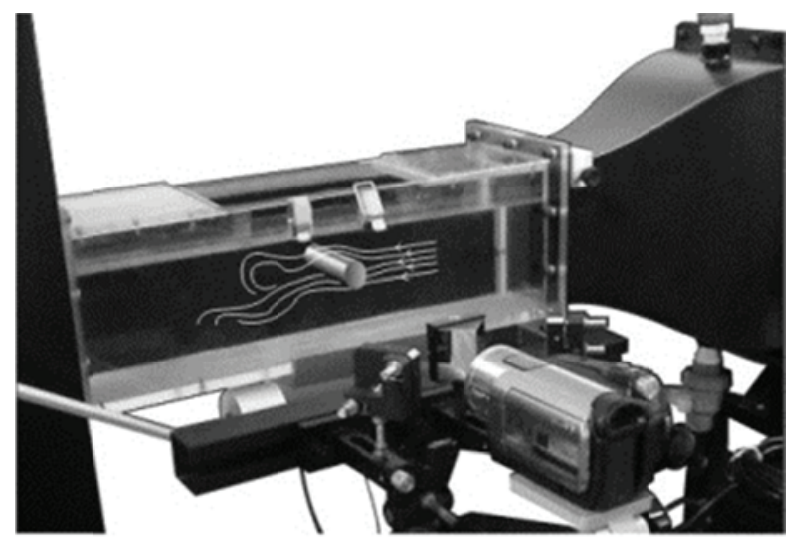

Na Figura 11, temos a reprodução de telas para as medições bidimensionais. Nessas telas, o campo vetorial e as linhas de corrente são sobrepostas à imagem de aquisição. No software S-PIV-3D, é possível a visualização sequencial de resultados com o acompanhamento do movimento das partículas, o controle de velocidade de exposição dos quadros, a visualização de quadros individuais e a extração de resultados numéricos a qualquer instante. Também são gerados gráficos interativos com a malha de vetores. A Figura 12 reproduz o resultado 
tridimensional com inclusão esquemática do modelo ensaiado. No resultado tridimensional, o plano xy é representado horizontalmente. Neste tipo de gráfico, é possível visualização sob qualquer ângulo, aproximação para observação de detalhes e separação das componentes dos vetores, entre outras funcionalidades.

Figura 11 - Telas de resultados: ensaio de um cilindro

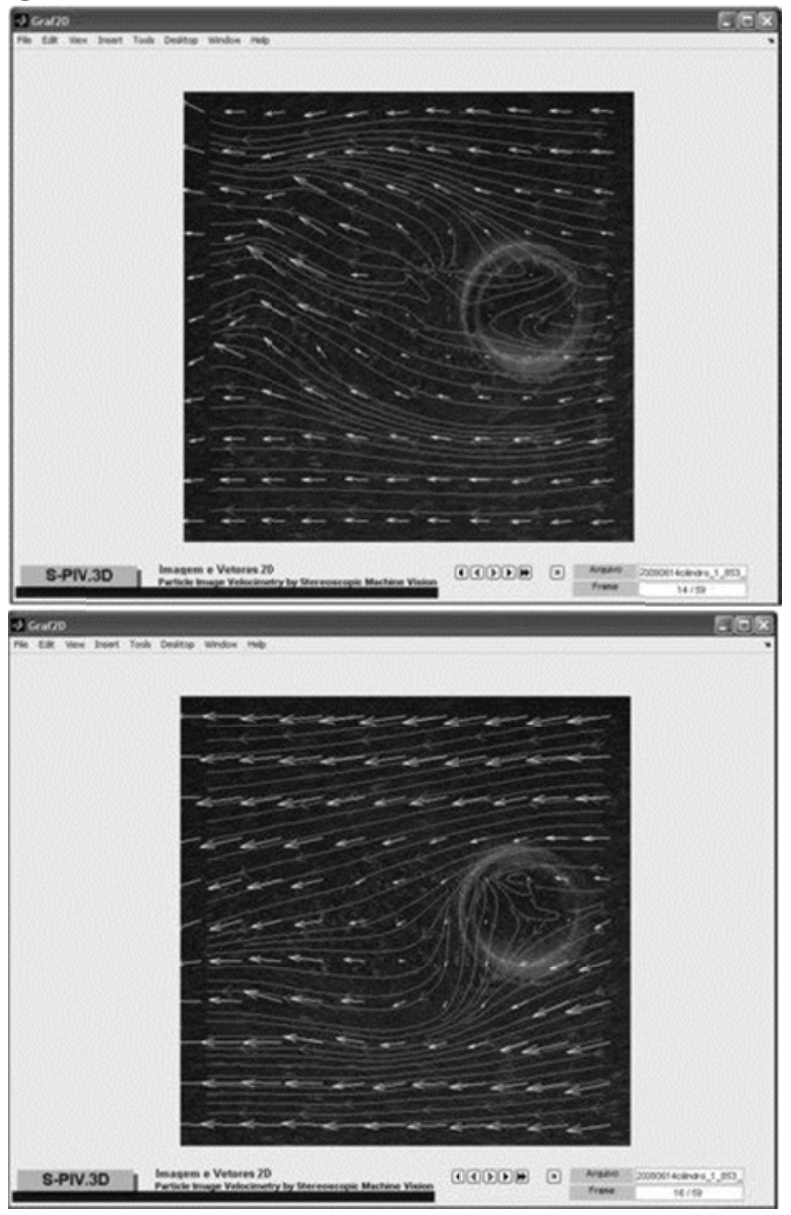

Figura 12 - Tela de resultados: modo tridimensional

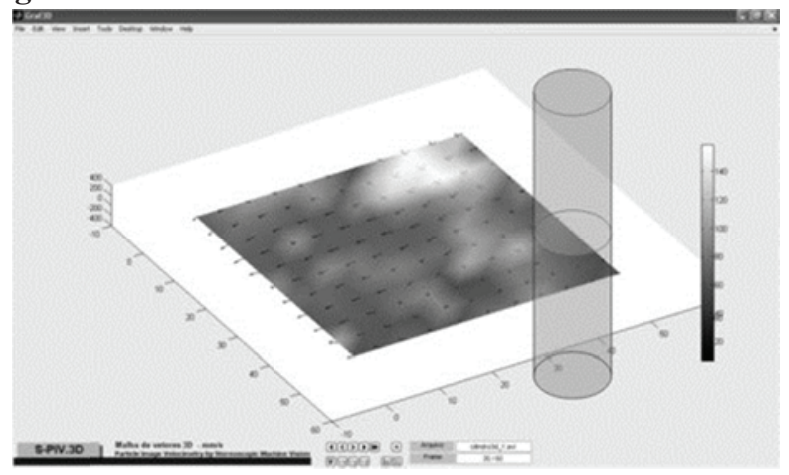

\subsection{Escoamento através de um orifício}

O ensaio foi realizado usando-se uma barra com orifício de seção quadrada com $15 \mathrm{~mm}$ de lado. A instalação deste ensaio está representada na Figura 13 e reproduções de resultados em modo de visualização bidimensional estão na Figura 14.

Figura 13 - Ensaio de escoamento através de um orifício

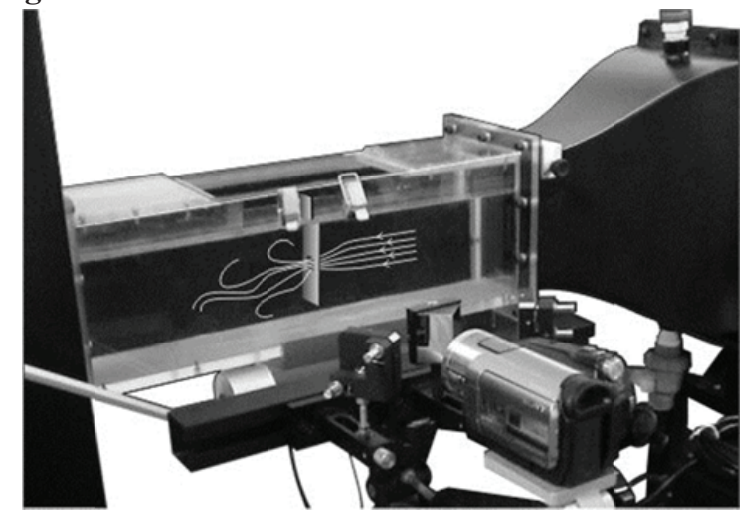

Figura 14 - Telas de resultados: ensaio de barra com orifício

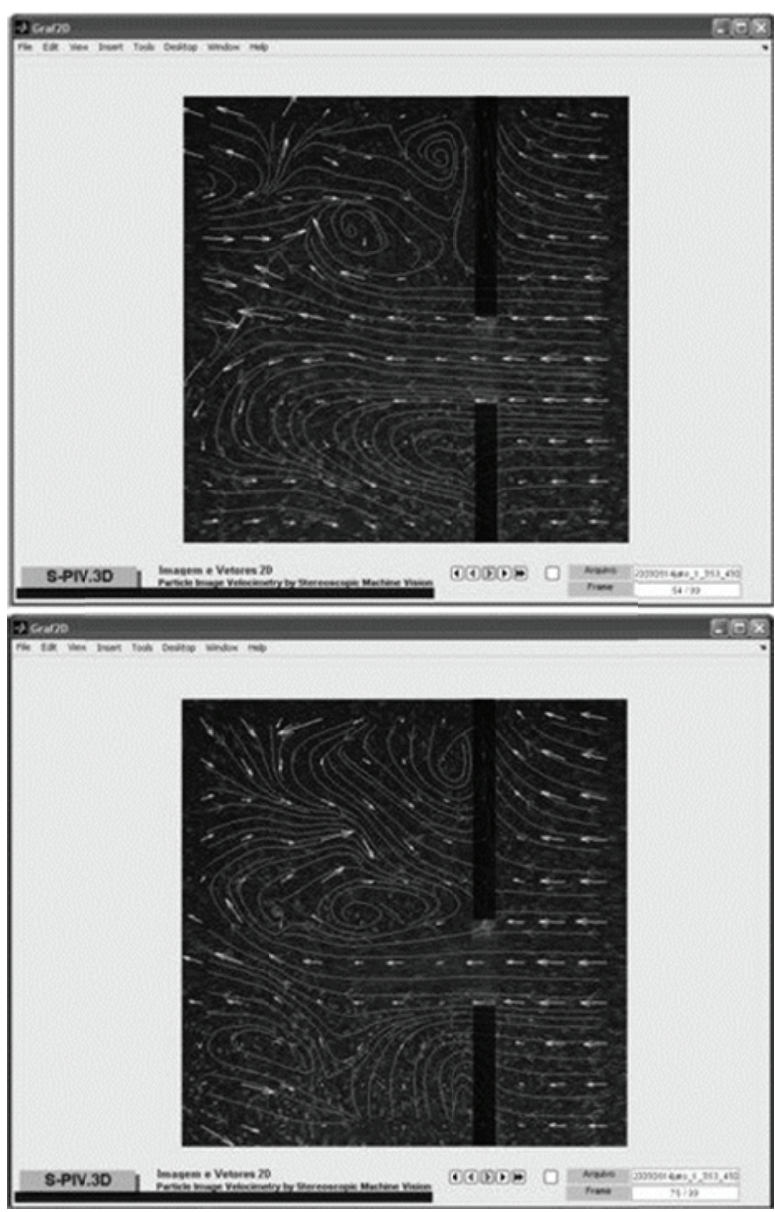




\subsection{Escoamento sob comporta de fundo}

O ensaio foi realizado usando-se uma barra com espaço inferior. A instalação deste ensaio está representada na Figura 15. No ensaio tridimensional, as imagens planas são mostradas simultaneamente, conforme a Figura 16. A Figura 17 é a reprodução da tela de resultados com o gráfico tridimensional.

Figura 15 - Ensaio de comporta de fundo

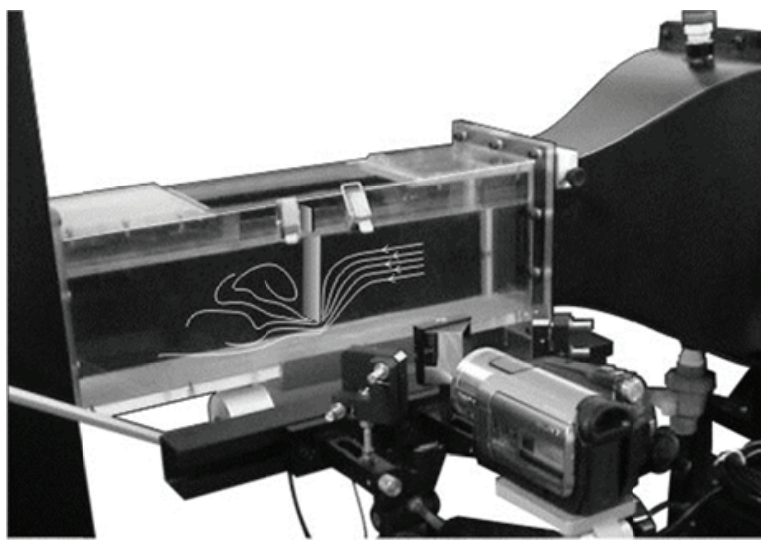

Figura 16 - Tela de resultados: ensaio tridimensional de comporta de fundo

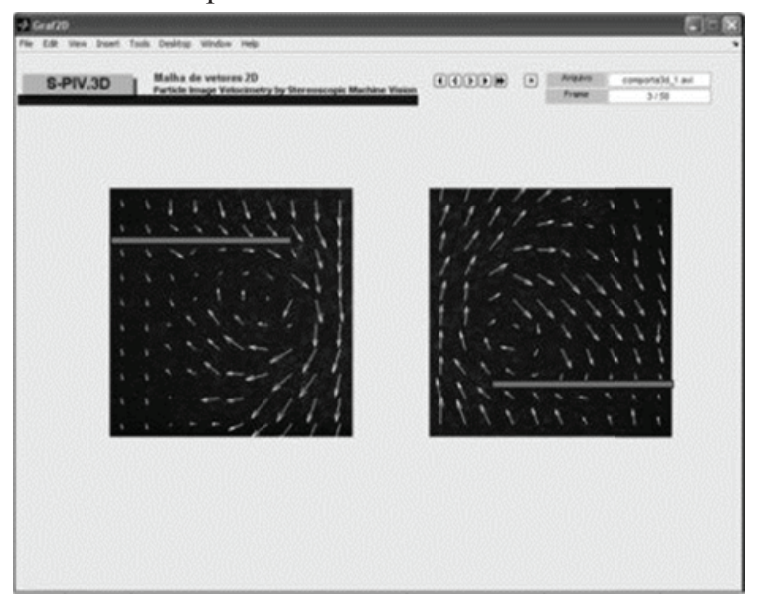

Figura 17 - Tela de resultados 3D

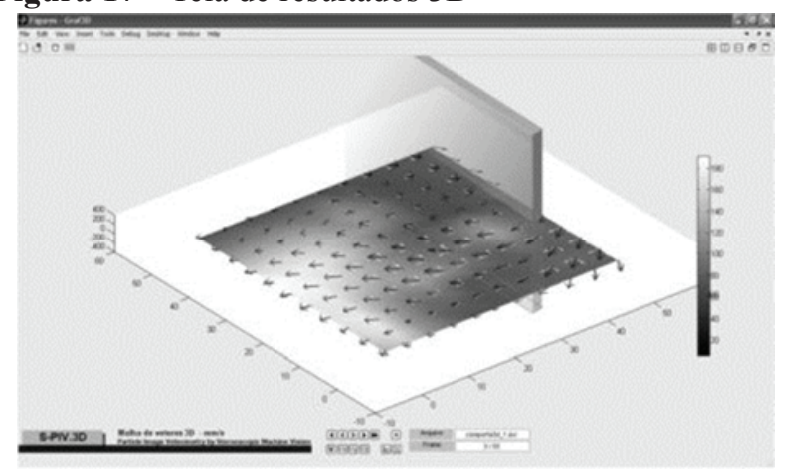

\section{CONCLUSÕES}

O desenvolvimento deste sistema teve um direcionamento para uma aplicação demonstrativa e com utilização de recursos visuais para sua instalação em laboratórios didáticos de ensino de engenharia. A utilização de dispositivos acessíveis integrados em uma metodologia específica garantiu a sua viabilidade como modelo para emprego de tecnologias atualizadas, na fronteira do conhecimento, nos laboratórios didáticos. Além dessa finalidade, pode-se notar a aplicabilidade com medições precisas para diagnóstico de escoamentos de baixo nível de turbulência.

Os autores agradecem o suporte do Conselho Nacional de Desenvolvimento Científico e Tecnológico (CNPq).

\section{REFERÊNCIAS}

CICCA, G. M.; IUSO, G.; SPAZZINI, P. G.; ONORATO, M. Particle image velocimetry investigation of a turbulent boundary layer manipulated by spanwise wall oscillations. J. of Fluid Mechanics, v. 467, p. 41-56, 2002.

DANTEC DYNAMICS Particle image velocimetry: institutional poster. Skovlunde, Dinamarca: Dantec Dynamics A/S., 2005.

ELSINGA, G. E.; OUDHEUSDEN, B. W. van; SCARANO, F. The effect of particle image blur on the correlation map and velocity measurement in PIV. Optical Engineering and Instrumentation, SPIE San Diego, 5880-37. 31 jul.-4 ago. 2005a.

for three-dimensional flows. Assessment of tomo-PIV SYMPOSIUM ON PARTICLE IMAGE VELOCIMETRY, 6. Anais... Pasadena, 21-23 set. 2005b.

JANSEN, K. D. Flow measurements. J. of Brazilian Society of Mechanics Science and Engineering, v. 26, n. 4, p. 400-19, 2004.

LAVISION FlowMaster Advanced PIV/ PTV. Systems for quantitative flow field analysis. Goettingen: LaVision GmbH, 2005.

RAFFEL, M.; WILLERT, C.; WERELEY, S.; KOMPENHANS, J. Particle image velocimetry: A pratical guide. 2. ed. Nova York: Springer Verlag, 2007.

OKAMOTO, K.; NISHIO, S.; KOBAYASHI, T.; SAGA, T.; TAKEHARA, K. Evaluation of the 3D-PIV standard images PIV-STD project. J. of Visualization, v. 3, n. 2, p. 115-23, 2000. 
PETRACCI, A.; DOOME, C.W.H.; WESTERWELL, J.; LECORDIER, B. Analysis of stereoscopic PIV measurements using synthetic PIV images. EUROPIV-2 Workshop, Zaragoza, 2003.

PU, Y.; MENG H. Four-dimensional dynamic flow measurement by holographic particle image velocimetry. Applied Optics, Washington, v. 44, n. 36, p. 7.697-708, 2005.

SENGUPTA, T. K.; LIM T. T.; SAJJAN, S. V.; GANESH, S.; SORIA, J. Accelerated flow past a symmetric aerofoil:experiments and computations. J. of Fluid Mechanics, v. 591, p. 255-88, 2007.

SMITH, B. L.; GLEZER, A. Jet vectoring using synthetic jets. J. of Fluid Mechanics, v. 458, p.1-34, 2002.

SZELIGA, M. R.; ROMA, W. N. L. Sistema de visão computacional para medição de turbulência e correlação com a reaeração de corpos d'água receptores. Revista Engenharia Sanitária e Ambiental, v. 9, n. 1, p. 30-44, jan.-mar. 2004.

; _ Machine Vision system for turbulence measurement and correlation with the rearation of water bodies: Computational issues. Cilamce 2006 - Iberian Latin American Congress in Computational Methods in Engineering, 27. Belém, 2006.

Estudos de reaeração com velocimetria por imagens de partículas: Sistema S-PIV-3D. Revista Engenharia Sanitária e Ambiental, v. 14, n. 4, p. 499-510, 2009. Doi: 10.1590/S1413-41522009000400010

TAO, B.; KATZ, J.; MENEVEAU, C. Statistical geometry of subgrid-scale stresses determined from holographic particle image velocimetry measurements. J. of Fluid Mechanics, v. 457, p. 35-78, 2002.

URBAN, W. D.; MUNGAL, M. G. Planar velocity measurements in compressible mixing layers. J. of Fluid Mechanics, v. 431, p. 189-222, 2001.

YANG, Y; ROCKWELL, D. Wave interaction with a vertical cylinder: spanwise flow patterns and loading. J. of Fluid Mechanics, v. 460, p. 93-129, 2002. 